\title{
Las tecnologías de la información y comunicación en psicoterapia en COVID-19
}

\section{Information and communication technologies in COVID-19 psychotherapy}

\author{
Gilda Moreno Proaño ${ }^{1}$ \\ https://orcid.org/0000-0002-5012-1391 \\ Universidad Tecnológica Indoamérica, Ecuador \\ Diego Porras ${ }^{2}$ \\ https://orcid.org/0000-0002-8801-257X \\ Fundación Children International, Ecuador
}

Recibido: 25-05-2020

Aceptado: 18-08-2020

\section{Cita Recomendada}

Moreno, G. \& Porras, D. (2020). Las tecnologias de la información y comunicación en psicoterapia en COVID-19. Hamut'ay, 7 (2), 58-63.

http://dx.doi.org/10.21503/hamu.v7i2.2133

\section{RESUMEN}

En el presente artículo se realiza una revisión bibliográfica acerca de las Tecnologías de la Información y Comunicación en la psicoterapia en Covid-19, la misma que tiene por objetivo analizar diferentes aspectos que conforman la estructura de una intervención psicoterapéutica, tales como, las herramientas tecnológicas utilizadas, la comunicación y la relación psicoterapéutica, la confidencialidad, los aspectos éticos intervinientes y las ventajas y desventajas de la modalidad virtual a la que tanto pacientes como terapeutas se han visto obligados a adaptarse por motivos del confinamiento en el mundo.

Palabras clave: matemáticas, COVID-19, psicoterapia, psicoterapia on line, teleasistencia psicológica, TIC.

\section{Abstract}

In this article is carried out a literature review about Information and Communication Technologies (ICTs) in COVID-19 psychotherapy, the main objective analyze different aspects that define structure psychotherapeutic intervention, such as, the technology tools used, communication and psychotherapeutic relationship, confidentiality, ethical intervening aspects and the advantages and disadvantages of the virtual modality that patients as therapists have been forced to adapt for reasons of confinement in the world.

\footnotetext{
1 Psicóloga Clínica, Psicoterapeuta. Docente de la Carrera de Psicología en Quito de la Universidad Tecnológica Indoamérica. Su línea principal de investigación es la Psicoterapia Cognitiva. Ha construido una metodología de atención psicológica con enfoque intercultural, para violencia intrafamiliar en poblaciones indígenas. E-mail: gyldymoreno@hotmail.com.

2 Psicólogo clínico, Psicoterapeuta, Magister en Planificación y Dirección Estratégica Actualmente se desempeña como Director de Programas en la Fundación Children International Experiencia en resolución de conflictos a través de herramientas de la psicología. Ha diseñado planes de acción para el desarrollo psíquico, emocional y profesional para adolescentes y jóvenes en situación de riesgo. E-mail: diegorporras@hotmail.com
} 
Keywords: COVID-19, psychotherapy, psychotherapy on line, psychological remote assistance

\section{INTRODUCCIÓN}

El Covid-19 aparece en Wuhan en el año 2019 y se convierte en una pandemia, provocando un alto impacto en cada una de las poblaciones, tanto a nivel de salud, familiar, educativo, económico y por supuesto, emocional, éste último de gran intensidad porque ha acarreado problemas relacionados principalmente con la aparición de síntomas depresivos, de ansiedad, irritabilidad, dificultades en el sueño, conflictos intrafamiliares y sociales, entre otros (Alvites-Huamaní, 2020); razones por las cuales, las personas han buscado ayuda psicológica.

La atención psicológica en el tiempo de confinamiento forzado, se ha manifestado con el uso de las Tecnologías de la Información y la Comunicación (TIC), a través de una cita virtual, utilizando herramientas óptimas para lograr interactuar con el paciente y obtener resultados exitosos similares a los que se alcanzan en la modalidad presencial.

Las herramientas tecnológicas se convierten en el recurso único para el abordaje de la problemática del paciente; sin embargo, no reemplaza a la estructura propia de una intervención psicoterapéutica, que se mantiene desde el establecimiento del rapport, el encuadre, las fases de la psicoterapia y el seguimiento al paciente.

Es trascendental que los profesionales de la salud mental estén capacitados en el uso y el proceso a seguir en una intervención en línea, puesto que así lo requiere la población en la actualidad, considerando la disposición ante la cual las nuevas generaciones hacen de la tecnología parte de su vida; por lo tanto, la adaptación a este medio se hace con mayor rapidez.

\section{MÉTODO}

Para el presente manuscrito se realizó una revisión bibliográfica minuciosa, de artículos científicos, documentos y tesis que aborden el tema de las TIC en psicoterapia, que reposan en buscadores como Google Scholar, SciElo, Redalyc, repositorios virtuales institucionales y Elsevier, desde el año 2015 hasta el 2020. Se usaron descriptores como: Las TIC en psicoterapia, tecnologías de la comunicación en psicología, teleasistencia psicológica, el rol de las TIC en psicoterapia y salud mental en Covid-19. La recopilación de la revisión bibliográfica se inició en junio del 2020 mientras se vivía la pandemia del Covid-19.

\section{Herramientas tecnológicas}

En el tiempo del Covid-19, la psicoterapia ha dado un giro total, se ha volcado a la virtualización; ante la cual, Lorenzo, Díaz \& Zaldívar, (2020) reflexionan en que son las Tecnologías de la Información las que prevalecen en la crisis, siendo éstas WhatsApp, Skype, Zoom, Facebook, entre las plataformas más usadas. Las herramientas tecnológicas mencionadas en psicoterapia no reemplazan a la tradicional, así como también a la evaluación, manejo y procesamiento de datos y asesoramiento (Distéfano et al., 2015) y se estima que en un futuro cercano las intervenciones terapéuticas telefónicas, videoconferencias y utilizando computadoras, irán en incremento.

Es importante resaltar que, en este análisis, los autores no se refieren a un cambio en la estructura de los procesos psicoterapéuticos, sino únicamente anotan el medio por el cual se brindará el servicio, no por esto se obviará los probables cambios que esta mediación producirá en la relación entre el terapeuta y el paciente. De igual manera, será motivo de reflexión el alcance de la psicoterapia mediatizada por computadoras u otro aparato similar, así como sus indicaciones y sus contraindicaciones.

Antes de la pandemia COVID-19 y del confinamiento que ésta obligó, trayendo el distanciamiento social como su principal componente, Bunge et al., (2015) afirman que la psicoterapia en línea, no reemplaza a la psicoterapia tradicional; es así que 
se convierten en un complemento que permite llegar a una población más extensa.

Es así, que se corrobora la afirmación realizada en el párrafo precedente; sin embargo, la realidad latente no permite que se vislumbre a la psicoterapia mediada por cualesquiera de los medios tecnológicos que hoy en día se dispone como un complemento a la psicoterapia tradicional, sino como única forma de ejecución de la misma. Esta inferencia lleva a dos cuestionamientos: ¿Qué fases de la psicoterapia son aquellas que se verán afectadas como consecuencia de la mediación de las TIC?; y, ¿Cuáles son las habilidades que el psicoterapeuta deberá adquirir o fortalecer como consecuencia de la mediación de las TIC dentro del proceso psicoterapéutico?

Sin duda alguna el día a día de la labor psicoterapéutica se encargará de resolver estas inquietudes, lo que a su vez propenderá mayor solvencia en el ejercicio profesional y, sin embargo, como investigadores se deberá prever estas nuevas variables para que su aparecimiento sorpresivo no vaya en detrimento de los pacientes.

Es importante mencionar a aquellas intervenciones psicoterapéuticas en las cuales interviene un sistema web y el paciente, ante lo cual, Barak, Klein \& Proudfoot (2009) señalan las intervenciones basadas en la Web o Web-Based interventions, mismas que hacen referencia a un sistema operado a través de una plataforma Web en la cual el paciente guía su proceso psicoterapéutico gracias a un programa prescriptivo que se encuentra en línea. Con este programa se pretende dar asistencia relacionada a la salud mental y a la salud en general. El programa contiene material que se enfoca en las áreas anteriormente mencionadas e incluye herramientas prácticas de auto aplicación, pretendiendo de esta manera ampliar la conciencia y comprensión por parte del usuario quien tendrá acceso a diferentes componentes interactivos que facilitan el proceso.

Es importante tener en cuenta que existen estas dos modalidades en los procesos psicoterapéuticos que utilizan herramientas tecnológicas, mismas que a pesar de no ser las únicas, ayudan a referenciar los grandes campos en los cuales se accionaría un proceso de psicoterapia; es así, que es posible discriminar qué tan eficientes pueden ser cada una de éstas modalidades de acuerdo a la especificidad de una patología, su intensidad y su etiología; así como también, ayudar a discernir la excelencia de la aplicabilidad de la herramienta desde el análisis del potencial usuario, discriminando datos como: edad, tipo de personalidad, familiaridad en la utilización de estas herramientas, entre otras.

Al hablar de tecnologías de vanguardia es inevitable referirse a la inteligencia artificial como un factor determinante en la utilización de herramientas tecnológicas para el proceso de psicoterapia. Es este sentido Rivera \& Sánchez (2016) mencionan conceptos como la cyberterapia para el tratamiento de fobias a través de la realidad virtual y una variedad de herramientas de la inteligencia virtual que permite intervenir en diferentes casos.

La autonomía que pretende dar la inteligencia artificial a los nuevos sistemas de gestión tecnológica, entre los que se incluirían los sistemas de gestión psicoterapéutica, modificaría el entendimiento de la relación entre el psicoterapeuta y el paciente, ya que a pesar de que la representación del psicoterapeuta seguiría existiendo como una simulación imperceptible pero informada, en la práctica será un algoritmo estructurado para cumplir esa finalidad, como se muestra en la figura 1.

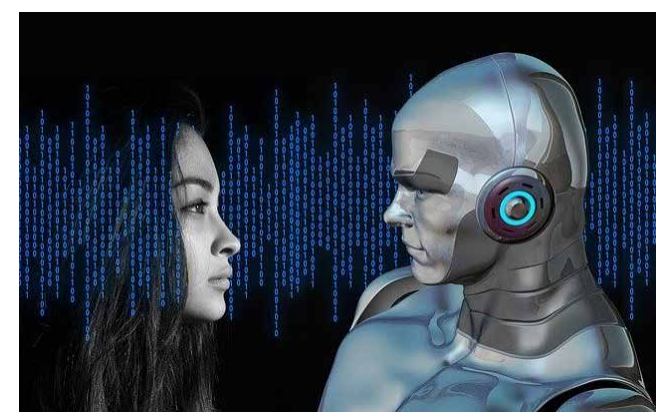

Figura 1. Binario código

Fuente: Pixabay, 2020

Las bases sobre las cuales se estructuraría la autonomía del sistema tecnológico que tiene como finalidad brindar sesiones psicoterapéuticas, dependen del sistema de aprendizaje con el que éste cuente, así como de la corriente psicoterapéutica con la que el sistema sea programado.

Sánchez (citado por Rivera, 2016) menciona que nunca un sistema con inteligencia artificial podrá reemplazar a un ser humano debido a las parti- 
cularidades propias del ser humano tales como: la creatividad, liderazgo, toma de decisiones y la resolución de conflictos; sin embargo, los sistemas de simulación y los algoritmos de aprendizaje sustentados en big data avanzan de manera exponencialmente rápida, lo que genera que la brecha que diferencia a una intervención humana con una simulada sea cada vez menos perceptible.

Otra forma de comunicación es a través de una línea telefónica de ayuda, cuya intervención según Valladares \& López (2020) tiene como propósito guiar al individuo a tomar decisiones acertadas y reducir las emociones negativas; así como también, identificar señales de alerta como ideas suicidas.

\section{La Comunicación y la Relación}

\section{Psicoterapéutica}

La relación en la atención psicoterapéutica en la cual participa un psicólogo, es un intercambio mutuo de aspectos objetivos y subjetivos. Las TIC median y promueven la reconfiguración constante y continua de los procesos de subjetividad, puesto que los hombres han incorporado y asimilado la tecnología como parte de su vida lo cual implica un cambio de paradigma de la forma de relacionarse y conformar la identidad mediados por la tecnología (Gil - Juárez, Vall-LLovera \& Feliu, 2010).

En la actualidad quienes acceden con mayor facilidad son los nacidos en una era tecnológica, que se adaptan fácilmente a las herramientas utilizadas en la intervención. En consecuencia, la construcción de una relación terapéutica es posible tanto a nivel presencial como virtual; sin embargo, cada una tiene sus especificidades.

Eiroá, Fernández \& Nieto (2017) sugieren algunos factores importantes que influyen positivamente en el desarrollo de una relación profesional entre el psicólogo y el paciente:

- Mantener una posición de igualdad con el paciente, puesto que este se encuentra en un estado de fragilidad por el momento de crisis que está viviendo; razón por la cual, no es momento de demostrar superioridad o directividad frente a él, es momento de presentar igualdad con el individuo que se encuentra atrás de la pantalla, como se evidencia en la figura 2 .

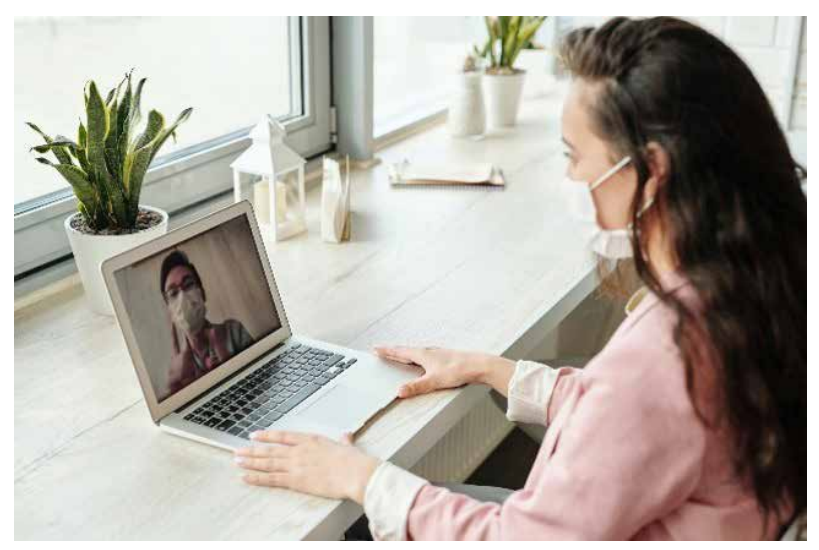

Figura 2. Adulto aislamiento

Fuente: Pexels, 2020

- Brindar una explicación clara en cada sesión psicológica de la metodología que se llevará a cabo en la misma, con el fin de proporcionar seguridad y confianza en el paciente.

- Mantener un ambiente de colaboración, en el cual existan espacios de diálogo para confrontar los aspectos negativos que podrían surgir en la terapia.

De la misma manera, el cambio radical se presenta en el uso de las tecnologías de la información y en los objetivos planteados, los mismos que se dirigen a la disminución de los síntomas a manera de primeros auxilios psicológicos. Así, la intervención psicológica en situaciones de crisis, motiva al paciente a la expresión de los sentimientos y experiencias internas, en relación con el evento que provocó el desajuste. Según Rodríguez et al., (2020). En efecto, el acompañamiento emocional es permanente y directo a través de la pantalla de un computador y recomiendan algunos aspectos que debe tener en cuenta el psicólogo al momento de utilizar una herramienta tecnológica para comunicarse con el paciente, figura 3.

Urzúa et al., (2020) revelan el modelo de intervención psicológica del West China Hospital que soporta la tecnología de Internet, redes de $4 \mathrm{G}$ o $5 \mathrm{G}$ o teléfonos inteligentes, como se muestra en la figura 3. Modelo que ha sido aplicado para los pacientes, familias y personal médico. 

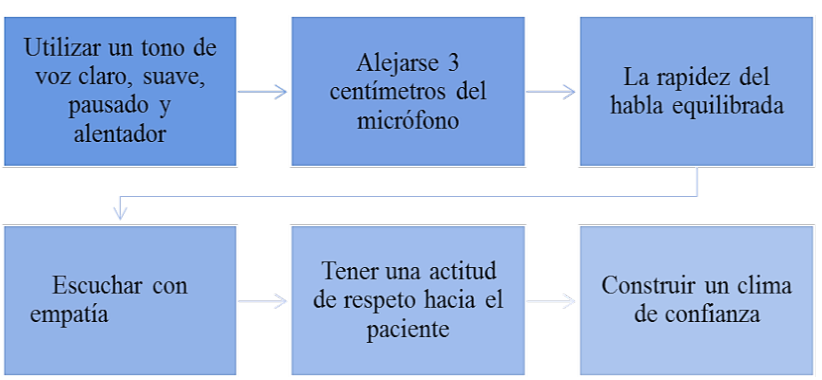

Tener una actitud de respeto hacia el paciente

Figura 3. Recomendaciones para comunicarse con el paciente al utilizar herramientas tecnológicas.

Fuente: Rodríguez et al., (2020)

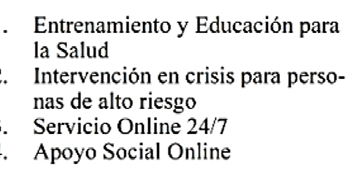

Figura 4. Modelo de intervención de crisis psicológica Fuente: Urzúa, et. al. 2020

El modelo de intervención en crisis, considera cuatro puntos importantes a tratar, el entrenamiento y educación para la salud, la intervención en crisis para personas de alto riesgo, servicio online $24 / 7$ y un apoyo social online.

\section{Principios Éticos y Confidencialidad}

Es importante para Mallen (2005) informar al paciente y aclarar la ruta que se deberá seguir en el caso de correr algún tipo de peligro él o los demás; así como también, ante una emergencia, indicar los datos informativos de una persona con quien se podrá comunicar el terapeuta. Información que quedará plasmada en un consentimiento informado.

La confidencialidad se convierte en el punto frágil de la intervención psicológica, por los recursos tecnológicos que se usan y queda en duda la seguridad de los datos proporcionados por el paciente, la dinámica de la intervención y la información que surge en la misma.

Fenichel (2011) considera que existe una extensa oferta de servicios que van desde los vocacionales, los que tratan los síntomas, los institucionales, sociales, problemas cotidianos, toma de decisiones, autoestima y habilidades; el terapeuta se convierte en un lazo para conectar directamente a una red de atención psicológica en línea.
Los pacientes tienen la posibilidad de escoger la modalidad de la psicoterapia, si es presencial o es en línea; a pesar que el confinamiento como exigencia de salud, ha disparado el porcentaje de atención psicológica virtual.

\section{Ventajas del Uso de las TIC en Psicoterapia}

- Para llegar a un diagnóstico psicológico, es necesario seguir el proceso que incluye el juicio clínico del psicólogo, considerando su experiencia, ideología y estilo. Roig-Fusté y Estallo (2018) afirma: "La máquina es imparcial y toma en cuenta sólo datos objetivos, evitando totalmente los prejuicios personales del clínico que pueden obstaculizar su capacidad de juicio o sesgar sus apreciaciones. (p.50).

- Se optimiza recursos, ya que tanto el psicólogo como el paciente no tienen que movilizarse para acudir a la cita establecida; más aún en el tiempo de confinamiento obligatorio. De igual manera, los horarios son más flexibles considerando la disposición de mayor tiempo, o a su vez, un tiempo prolongado.

- En la intervención psicológica en línea se facilita la lectura de documentos, libros, guías, artículos que ayuden al paciente en su tratamiento, los mismos que son distribuidos por el psicólogo de manera rápida.

\section{Conclusiones}

Según explica Roussos (2018) aún no existe una capacitación adecuada sobre el uso de las TIC y desencadena prácticas iatrogénicas que perjudican al paciente, las mismas que están directamente relacionadas con la confidencialidad, seguridad, aspectos éticos, rapport y encuadre omitidos.

Respecto al enfoque psicoterapéutico escogido por el psicoterapeuta, Labrador et al., (2003) manifiestan que no todos los tratamientos psicológicos han sido sometidos a prueba, el más investigado ha sido el enfoque cognitivo-conductual que es eficaz en algunas situaciones importantes del paciente; siendo así, también en el que mejor se adaptan las técnicas a aplicar en a la atención psicológica virtual. 
Las terapias psicoanalíticas, psicodinámicas, existenciales y sistémicas, no han sido suficientemente investigadas, con el fin de determinar su eficacia; por lo tanto, es un riesgo experimentar desde la virtualidad.

Desde nuestro punto de vista, en situaciones como, por ejemplo: crisis, ideas suicidas, trastornos alimenticios, violencia, abuso sexual, es mejor derivar a una modalidad presencial; sin embargo; en tiempos de Covid-19 se deben tomar estrictas medidas de bioseguridad para provocar dicho encuentro.

Durante la pandemia existe una sobre oferta de servicios psicológicos en línea, algunos de ellos sin una estructura sólida que pueden generar perjuicios en la comunidad.

Para Urzúa, et al. (2020) se deben incorporar los conocimientos en psicología ya adquiridos a las nuevas tecnologías, considerando que los teléfonos y el internet son buenos mecanismos para llegar a la población con un tratamiento psicológico; sin embargo, el contenido será científico y no una sobrecarga de tecnología sin sentido social.

\section{REFERENCIAS BIBLIOGRÁFICAS}

Alvites-Huamaní, C. (2020). Covid-19: Pandemia que impacta en los estados de Ánimo. CienciAmérica, 9 (2). https://doi.org/10.33210/ca.v9i2.327

Barak, A., Klein, B., \& Proudfoot, J. G. (2009). Defining internet-supported therapeutic interventions. Annals of behavioral medicine, 38(1), 4-17. https://doi.org/10.1007/ s12160-009-9130-7

Distéfano, M. J., Mongelo, M. C., OConor, J., \& Lamas, M. C. (2015). Psicoterapia y tecnología: implicancias y desafíos en la inserción de recursos innovadores en la práctica clínica argentina. Revista electrónica de psicología Iztacala, 18(4), 1342-1362.

Eiroá, F.; Fernández, M. y Nieto R. (2017). Formulación y tratamiento psicológico en el siglo XXI. Recuperado de http://www.digitaliapublishing.com.indoamerica.idm. oclc.org/a/47352/formulacion-y-tratamiento-psicologico-en-el-siglo-xxi

Fenichel, M. (2011). Comportamiento en línea, comunicación y experiencia. En asesoramiento en línea (pp. 3-20). Prensa Académica.

Gil-Juárez, Adriana, Vall-llovera, Montse, \& Feliu, Joel. (2010). Consumo de TIC y Subjetividades Emergentes:
¿Problemas nuevos? Psychosocial Intervention, 19(1), 19-26. https://doi.org/10.5093/in2010v19n1a4

Labrador, F. J., Vallejo, M. Á., Matellanes, M., Echeburúa, E., Bados, A., \& Fernández-Montalvo, J. (2003). La eficacia de los tratamientos psicológicos. Infocop, 84.

Mallen, M., Vogel, D. y Rochlen, A. (2005). Los aspectos prácticos de la asesoría en línea: ética, capacitación, tecnología y competencia. El psicólogo consejero, 33 (6), 776-818. https://doi.org/10.1177/0011000005278625

Rivera Estrada, J. E., \& Sánchez Salazar, D. V. (2016). Inteligencia artificial: ¿reemplazando al humano en la psicoterapia? https://doi.org/10.18566/escr.v24n53.a02

Roig-Fusté, J.; Estallo, J. (2018). La informática aplicada a la psicología.. https://doi.org/10.2307/j.ctvh1dnhh

Roussos, A. J., \& Braun, M. (2018). Psicoterapia y tecnologías de información y comunicación. Psicoterapia 2.0. Enciclopedia Argentina de Salud Mental.

Roussos A. (2018) Cuando el diván no es el mismo. La irrupción de las tecnologías de información y comunicación (TIC) en la psicoterapia. Rev. Bras. psicoter.;20(3):229-240 https://doi.org/10.5935/2318-0404.20180016

Lorenzo Ruiz, A., Díaz Arcaño, K., \& Zaldívar Pérez, D. (2020). La psicología como ciencia en el afrontamiento a la COVID-19: apuntes generales. Anales De La Academia De Ciencias De Cuba, 10(2), https://doi.org/10.37226/rcp. v4i2.4815

Urzúa, Alfonso, Vera-Villarroel, Pablo, Caqueo-Urízar, Alejandra, \& Polanco-Carrasco, Roberto. (2020). La Psicología en la prevención y manejo del COVID-19. Aportes desde la evidencia inicial. Terapia psicológica, 38(1), 103-118. https://doi.org/10.4067/S0718-48082020000100103

Valladares González, Anais Marta, \& López Angulo, Laura Magda. (2020). Línea CONVIDA. Propuesta de guía práctica psicológica ante la situación emergente de la COVID 19. Cienfuegos, 2020. MediSur, 18(3), 416-430. Recuperado de http://scielo.sld.cu/scielo.php?script=sci_arttext\&pi$\mathrm{d}=$ S1727-897X2020000300416\&lng=es\&tlng=es. 